\title{
COLLINEATIONS IN A FINITE PROJECTIVE GEOMETRY*
}

BY

\section{OSWALD VEBLEN}

In the paper by Mr. Busser and myself on Finite Projective Geometries it was shown $\dagger$ that in a $P G\left(k, p^{n}\right)$ any transformation of the form

$$
\frac{x_{i}^{\prime}}{x_{k+1}^{\prime}}=\frac{a_{i 1} x_{1}^{p m}+a_{i 2} x_{2}^{p^{m}}+\cdots+a_{i k+1} x_{k+1}^{p^{m}}}{a_{k+11} x_{1}^{p^{m}}+a_{k+12} x_{2}^{p^{m}}+\cdots+a_{k+1 k+1} x_{k+1}^{p^{m}}} \quad(i=1, \cdots, k),
$$

where $m$ is zero or an integer less than $n$, is a collineation. As a result of Dr. Levi's article $\ddagger$ it is possible to prove the converse proposition, namely, that every collineation in $P G\left(k, p^{n}\right)$ is of type (1). The following argument connects this theorem directly with our former article. It will be sufficient to give the argument for the case, $k=2$.

A projective collineation, $\S$ or, in other words, a linear transformation, is determined by the quadrangle into which it transforms the quadrangle $\left(\begin{array}{lll}0 & 0 & 1\end{array}\right)$, (1 111$),\left(\begin{array}{lll}0 & 1 & 1\end{array}\right),\left(\begin{array}{lll}1 & 0 & 1\end{array}\right)$. It follows that an arbitrary collineation is the product of a projective collineation by a collineation which leaves these four points invariant. The latter type of collineation may be called antiprojective, in the language of SEGRE. $\|$ Its existence is proved by the existence of transformations of type (1) where $m \neq 0$.

An antiprojectivity, by its definition, leaves invariant the points of the $x_{1}$ axis for which $x_{1} / x_{3}$ is 0,1 , or $\infty$, and, therefore, all points of the chain determined by these three, namely, all points whose coördinates are integers modulo $p$. From the quadrangle-construction for addition and multiplication and the fact that a collineation transforms a complete quadrangle into a complete quadrangle it follows that if three points of the $x_{1}$ axis for which $x_{1} / x_{3}$ is $a, b$, and $c$ respectively are so related that

* Presented to the Society December 28, 1906. Received for publication November 16, 1906.

† These Transactions, vol. 7 (1906), p. 252.

$\ddagger$ Page 354 of this number of the Transactions. To our former list of works relating to finite geometries we add references to this paper of LEVI, Geometrie proiettive di congruenza $e$ geometrie proiettive finite, and to an earlier paper, Fondamenti della metrica proiettiva, Memorie della R. Accademia delle Scienze di Torino, ser. 2, vol. 54 (1904), and also to a book by G. ARnoux, Arithmétique Graphique. Les espaces arithmétiques hypermagiques, Paris, 1894. ARsoux applied his "arithmetic spaces," which may be identified with our $E G(k, p)$, to construction of magic squares.

§ These Transactions, vol. 7 (1906), p. 253.

॥ Cf. LEvi's remarks on page 357. 


$$
a+b=c \quad \text { or } \quad a b=c,
$$

then the points, with coördinates $\phi(a), \phi(b), \phi(c)$, into which they are transformed are so related that

$$
\phi(a)+\phi(b)=\phi(c) \quad \text { or } \quad \phi(a) \phi(b)=\phi(c) .
$$

In other words the transformation of the points of the $x_{1}$ axis effected by an antiprojectivity is subject to the conditions : *

$$
\begin{gathered}
\phi(a)+\phi(b)=\phi(a+b) ; \\
\phi(a) \cdot \phi(b)=\phi(a b) ; \\
\phi(0)=0, \quad \phi(1)=1, \quad \phi(\infty)=\infty .
\end{gathered}
$$

If $e$ is a primitive root of the $G F\left[p^{n}\right]$ then every mark of the field may be written in the form

But by (3)$$
e^{k} \text {. }
$$$$
\phi\left(e^{k}\right)=[\phi(e)]^{k} .
$$

Hence if $\phi(e)=e^{m}$, then $\phi\left(e^{k}\right)=e^{m k}=\left(e^{k}\right)^{m}$ and

$$
\phi(x)=x^{m} \text {. }
$$

If $k$ is a primitive root of the $G F[p]$ then

$$
\phi(k)=k .
$$

Hence $m$ must be of the form $p^{l} \dagger$ and therefore, as a transformation of the points of the $x_{1}$ axis, an anti-projectivity is given by the equation

$$
\frac{x_{1}^{\prime}}{x_{3}^{\prime}}=\frac{x_{1}^{p l}}{x_{3}^{p l}}
$$

and the number of antiprojectivities, exclusive of the identity, is $n-1$.

An antiprojectivity is, however, completely determined by the permutation it effects among the points of the $x_{1}$-axis. For by projection from the center $\left(\begin{array}{lll}1 & 0 & 1\end{array}\right)$ this permutation determines the only possible permutation of points on the fixed line $x_{1}=x_{2}$. But a collineation is completely determined by the

* This derivation of (2) and (3) applies to any desarguesian geometry, whereas the proof of (3) given by LeVr assumes the commutativity of multiplication. This is because Levi defines an antiprojectivity of a line as a transformation which changes harmonic throws into harmonic throws. The two definitions have not been proved identical in cases where multiplication is non-commutative. DARBoux's proof of the fundamental theorem of projective geometry amounts to showing that conditions (2), (3), (4) are satisfied in the case of ordinary number only by the identical transformation.

† Similarly if the marks of any $G F\left[p^{\nu}\right]$ included in the $G F\left[p^{n}\right]$ are required to be invariant, $\phi(x)=x\left(p^{\nu}\right)^{l}$. From this consideration are derived the results of the last section of Dr. Levi's paper. 
permutations of the points of two fixed lines. There are thus $n-1$ antiprojectivities of the plane. Since the following are evidently $n-1$ distinct antiprojectivities they must therefore be all the antiprojectivities of the plane:

$$
\frac{x_{1}^{\prime}}{x_{3}^{\prime}}=\frac{x_{1}^{p l}}{x_{3}^{p l}}, \quad \frac{x_{2}^{\prime}}{x_{3}^{\prime}}=\frac{x_{2}^{p^{l}}}{x_{3}^{p^{l}}} .
$$

The most general collineation is now evidently a product of $(5)$ by the most general linear fractional transformation and that product is of type (1). It follows as a corollary that the order of the collineation group of $P G\left(k, p^{n}\right)$ is the order of the linear fractional group $L F\left(k+1, p^{n}\right)$ multiplied by $n$.

Princeton, N. J.,

November 13, 1906. 